\title{
LOCAL AND 2-LOCAL ISOMETRIES BETWEEN ABSOLUTELY CONTINUOUS FUNCTION SPACES
}

\author{
MaLiheH Hosseini AND JUAN J. FONT
}

Abstract. In this paper we give a complete description of local and 2-local isometries defined between spaces of scalar-valued absolutely continuous functions on arbitrary (not necessarily compact) subsets of the real line with at least two points.

Mathematics subject classification (2020): Primary 47B38; Secondary 46J10, 47B33.

Keywords and phrases: Linear surjective isometry, absolutely continuous functions, local isometry, 2-local isometry.

\section{REFERENCES}

[1] H. Al-Halees, R. J. Fleming, On 2-local isometries on continuous vector-valued function spaces, J. Math. Anal. Appl. 354 (2009), 70-77.

[2] F. CABello SÁnCHEZ, Local isometries on spaces of continuous functions, Math. Z. 251 (2005), $735-749$.

[3] R. J. Fleming, J. E. Jamison, Isometries on Banach Spaces: Function Spaces, Chapman Hall/CRC Monogr. Surv. Pure Appl. Math., 129, Chapman Hall/CRC, Boca Raton, 2003.

[4] M. GYÖRY, 2-local isometries of $C_{0}(X)$, Acta Sci. Math. (Szeged) 67 (2001), 735-746.

[5] O. Hatori, T. Miura, H. OKA, H. TAKAGI, 2 -local isometries and 2-local automorphisms on uniform algebras, Int. Math. Forum 50 (2007), 2491-2502.

[6] M. HossEINI, Algebraic reflexivity of sets of bounded linear operators on absolutely continuous function spaces, Oper. Matrices 13 (2019), 887-905.

[7] M. Hosseini, 2-Local isometries between spaces of functions of bounded variation, Positivity 24 (2020), 1101-1109.

[8] M. Hosseini, J. J. FonT, Isometries on spaces of absolutely continuous functions in a noncompact framework, J. Math. Anal. Appl. 487 (2020), 123962.

[9] K. Jarosz, T. S. S. R. K. RAO, Local isometries of function spaces, Math. Z. 243 (2003), 449-469.

[10] A. JimÉneZ-VARGAS, M. VILLEGAS-VALLECILlos, 2-iso-reflexivity of pointed Lipschitz spaces, J. Math. Anal. Appl. 491 (2020), https://doi.org/10.1016/j.jmaa.2020.124359.

[11] A. JimÉneZ-VARGAS, M. Villegas-VALLECiLlos, 2-local isometries on spaces of Lipschitz functions, Canad. Math. Bull. 54 (2011), 680-692.

[12] L. Li, A. M. Peralta, L. WANG, Y.-S. WANG, Weak-2-local isometries on uniform algebras and Lipschitz algebras, Publ. Mat. 63 (2019) 241-264.

[13] L. Molnár, Selected Preserver Problems on Algebraic Structures of Linear Operators and on Function Spaces, Lecture Notes in Mathematics, 1895, Springer-Verlag, Berlin, 2007.

[14] L. MOLnÁR, B. ZALAR, Reflexivity of the group of surjective isometries on some Banach spaces, Proc. Edinb. Math. Soc. 42 (1999), 17-36.

[15] S. OI, Algebraic reflexivity of isometry groups of algebras of Lipschitz maps, Linear Algebra Appl. 566 (2019), 167-182.

[16] P. ŠEmRL, Local automorphisms and derivations on B(H), Proc. Amer. Math. Soc. 125 (1997), $2677-$ 2680. 